\title{
Numerical Study of the Acoustic Break-up of a Liquid Droplet
}

\author{
Michael B. Kuhn*, Olivier Desjardins \\ Sibley School of Mechanical and Aerospace Engineering, Cornell University, Ithaca, NY, \\ U.S.A. \\ *Corresponding author email: mbk225@cornell.edu
}

\begin{abstract}
Combustion instabilities represent a major design challenge for aerospace engines. In liquidfueled systems, such instabilities can lead to high frequency coupling of the atomization, combustion, and acoustics processes. This class of instabilities tends to be the most dangerous and the least understood. Additionally, there exists significant interest in acoustic enhancement of fuel atomization. To contribute to a fundamental understanding of the interactions between surface tension and acoustic processes present in these flows, we characterize the acoustic break-up of a liquid droplet via high-fidelity numerical simulations. Placing a quiescent droplet in a forced acoustic field, we probe the effects of different flow parameters, including wave frequency, droplet position, and background turbulence. To perform these simulations, we employ an all-Mach, multiphase flow solver, which features a geometric, semi-Lagrangian transport method and pressure projection scheme. Through studying droplet break-up in this context, we provide physical insight as well as details for future models of acoustically enhanced atomization.
\end{abstract}

\section{Keywords}

acoustics, atomization, compressible multiphase flow

\section{Introduction}

In liquid-fueled rocket engines, the coupling of heat release, acoustic waves, and the hydrodynamic flow field contributes to combustion instabilities that can impact performance and may lead to system destruction [1]. The atomization process of the liquid fuel is integral to the relationship between these processes, with the fuel spray responding to the acoustic field in the engine chamber while contributing to the hydrodynamic flow and heat release characteristics [2]. Although the large-scale behavior of these instabilities has been widely studied, the local behavior of the atomization in the presence of acoustics needs to be further understood. In addition to the mitigation of combustion instabilities, the interaction between sound waves and droplet breakup is also relevant for fuel injection strategies in general. Introducing acoustic energy to an injection system can enhance atomization [3], and acoustic systems provide opportunity for control of atomization processes.

In general, the significantly higher inertia of liquid structures prevents an instantaneous response to acoustic perturbations in the surrounding gas phase. However, high frequency signals lead to nonlinear radiative pressures surrounding obstacles within the acoustic field, exerting a consistent time-averaged force on such obstacles [4]. This effect can serve to reshape and distort liquid structures as well as alter the trajectories of droplets [2]. Moreover, when a liquid structure becomes sufficiently thin, transverse acoustic excitation can induce instabilities that result in atomization [5].

Experimental studies performed by Baillot et al. [2] and Ficuciello et al. [6] have investigated the effect of high frequency acoustic fields on atomizing jets, as well as simulations such as those performed by Natarajan and Desjardins [7]. In the presence of a transverse acoustic field, a liquid jet can undergo a flattening effect that spatially rearranges the resulting spray and influences the droplet size distribution [2]. 
Acoustically induced atomization has also been studied in the context of a single drop exposed to a high-frequency acoustic field. Often, these experiments are relevant to the topic of acoustically levitated droplets and seek to elucidate the stability limits of such droplets. Lee et al. develop a semi-analytical framework to predict the stability limit of individual droplets [8], informing further experimental studies. The viscosity of the liquid can significantly affect the nature of droplet stability, damping capillary waves and preventing atomization, sometimes leading to film buckling and bubble formation instead [5, 9]. Yarin et al. develop a calculation, based on the boundary element method, to predict the shape of levitated droplets and the associated acoustic radiation pressure [10]. This framework is expanded to analyze evaporating droplets, and the phenomenon of acoustic streaming is described in detail [11].

To focus on the physics of acoustically-driven atomization in a simpler setting, we simulate the atomization of a single droplet. We first validate our numerical approach by comparing with experiments of levitating droplets. Then, we investigate the role of acoustic frequency, initial location, and background turbulence in atomization. Through the use of simulations, we are able to study scenarios that are less accessible to earth-based experiments. Ordinarily, the influence of gravity demands the liquid droplet be placed near an equilibrium position, but in numerical experiments, removing the effect of gravity allows the the droplet to be freely positioned along the acoustic wave and prevents gravity from interfering with the resulting droplet dynamics. From the simulation results, we identify relationships between droplet dynamics and the flow parameters and discuss how these behaviors impact atomization.

\section{Governing equations and methods}

We solve the two-phase compressible Navier-Stokes equations using a six-equation model from [12] that assumes a continuous velocity field and enforces mechanical equilibrium via pressure relaxation. Adding terms to incorporate surface tension and viscous effects, the governing equations are

$$
\begin{array}{rlrl}
\frac{\partial \alpha_{s}}{\partial t}+\boldsymbol{u} \cdot \nabla \alpha_{s} & & \eta\left(p_{s}-p_{s^{\prime}}-[p]_{s s^{\prime}}\right) \\
\frac{\partial \alpha_{s} \rho_{s}}{\partial t}+\nabla \cdot\left(\alpha_{s} \rho_{s} \boldsymbol{u}\right) & & 0 \\
\frac{\partial \rho \boldsymbol{u}}{\partial t}+\nabla \cdot(\rho \boldsymbol{u} \otimes \boldsymbol{u})+\nabla p & & \nabla \cdot \boldsymbol{\tau}+\boldsymbol{f} \\
\frac{\partial \alpha_{s} \rho_{s} E_{s}}{\partial t}+\nabla \cdot\left(\alpha_{s} \rho_{s} E_{s} \boldsymbol{u}\right)+\frac{\alpha_{s} \rho_{s}}{\rho} \boldsymbol{u} \cdot \nabla p+\alpha_{s} p_{s} \nabla \cdot \boldsymbol{u} & =\frac{\alpha_{s} \rho_{s}}{\rho} \boldsymbol{u} \cdot(\nabla \cdot \boldsymbol{\tau})+\alpha_{s} \boldsymbol{\tau}_{s}: \nabla \boldsymbol{u} \\
& +\frac{\alpha_{s} \rho_{s}}{\rho} \boldsymbol{u} \cdot \boldsymbol{f}-\eta p_{I}\left(p_{s}-p_{s^{\prime}}-[p]_{s s^{\prime}}\right),
\end{array}
$$

where $s$ denotes one of the fluid phases and $s^{\prime}$ is the other. The evolution of the volume fraction, $\alpha_{s}$, is calculated for a single phase, the evolution of the density, $\rho_{s}$, and the total energy, $\rho_{s} E_{s}$, is calculated for each individual phase, and the momentum, $\rho \boldsymbol{u}$, is calculated in a single equation representing the combined momentum. The pressure relaxation term is set to $\eta \rightarrow \infty$, which enforces instantaneous mechanical equilibrium each step. This equilibrium process also considers the pressure jump between phases, $[p]_{s s^{\prime}}$, which is equal to the surface tension coefficient multiplied by the curvature, $\sigma \kappa$. The mixture pressure is defined as $p=\alpha_{1} p_{1}+\alpha_{2} p_{2}$ and the mixture density is defined as $\rho=\alpha_{1} \rho_{1}+\alpha_{2} \rho_{2}$. The body forces, including surface tension, are included in $\boldsymbol{f}$, and the viscous stress tensor is $\boldsymbol{\tau}=\mu\left(\nabla \boldsymbol{u}+(\nabla \boldsymbol{u})^{T}-(2 / 3) \nabla \cdot \boldsymbol{u} \boldsymbol{I}\right)$. We assume $\tau_{s}=\tau$ and calculate the viscosity, $\mu$, by harmonic averaging between the phases. We solve these equations using the algorithm described in [13]. This consists of a hybrid advection scheme that combines a geometric semi-Lagrangian method adapted from $[14,15]$ with a centered scheme adapted from [16]. A planar interface is used to distinguish between phases within cells (PLIC) [17], which is reconstructed according to ELVIRA [18]. Implementation of the 
geometric operations in advection and the interface reconstruction is facilitated by the use of the Interface Reconstruction Library (IRL) [19, 20, 21]. An all-Mach projection scheme, inspired by [22], is used to determine the pressure field. This involves solving a Helmholtz equation derived from the governing equation for pressure,

$$
\frac{\partial p_{s}}{\partial t}+\boldsymbol{u} \cdot \nabla p_{s}=\frac{1}{\rho_{s}} \frac{\partial p_{s}}{\partial e_{s}} \boldsymbol{\tau}_{s} \cdot \nabla \boldsymbol{u}-\rho_{s} c_{s}^{2} \nabla \cdot \boldsymbol{u} .
$$

Overall, this numerical framework is conservative, robust, minimally dissipative, and capable of capturing compressible and multiphase flow dynamics.

\section{Validation}

As validation of our numerical approach for acoustically-driven multiphase problems, we replicate experiments by Anilkumar et al. [23]. Specifically, we simulate the case where $k R_{s}=0.58$, where $k$ is the wavenumber of the acoustic signal and $R_{s}$ is the radius of the initially spherical droplet. In these experiments, the frequency of the signal is $f=21.76 \mathrm{kHz}$. The experiments are performed at room temperature and atmospheric pressure with water droplets.

To model the liquid phase, we use the stiffened gas equation of state,

$$
p=(\gamma-1) \rho e+p_{\infty},
$$

with $\gamma=4.4$ and $p_{\infty}=6 \times 10^{8} \mathrm{~Pa}$. The dynamic viscosity is $\mu=900 \mu \mathrm{Pa} \mathrm{s}$, the initial density is $\rho=998 \mathrm{~kg} / \mathrm{m}^{3}$, and the surface tension coefficient is $\sigma=90 \mathrm{mN} / \mathrm{m}$. The gas phase is modeled with the ideal gas equation of state,

$$
p=(\gamma-1) \rho e,
$$

with $\gamma=1.4$. The dynamic viscosity is $\mu=18.13 \mu \mathrm{Pa}$ s and the initial density is $\rho=1.2 \mathrm{~kg} / \mathrm{m}^{3}$. The acoustic field is created through sponge zones at the upper and lower boundaries of the domain. These use a spatially and temporally varying source term to nudge the flow toward a standing wave solution. Within a volumetric region at each boundary, the sponge term is given

$$
\frac{\partial \boldsymbol{Q}}{\partial t}=\mathrm{RHS}-C_{\text {sponge }} \xi^{2}\left(\boldsymbol{Q}-\boldsymbol{Q}_{\text {target }}\right),
$$

where RHS is the right-hand side of the Navier-Stokes equations, $C_{\text {sponge }}$ is the sponge amplitude, $\xi$ is a normalized sponge coordinate, and $Q_{\text {target }}$ is the target solution. The normalized coordinate $\xi$ is equal to 1 at the edge of the sponge closest to the domain boundary and 0 at the opposite edge, closest to the center of the domain. The target solution in our simulations is a standing wave, which follows the equations

$$
\left(p^{\prime}, \rho^{\prime}, v^{\prime}\right)=\left(A \sin (k y) \cos (2 \pi f t), p^{\prime} / c_{0}^{2},-A \cos (k y) \sin (2 \pi f t) /\left(\rho_{0} c_{0}\right)\right),
$$

leading to the target solution of

$$
\boldsymbol{Q}_{\text {target }}=\left[\begin{array}{c}
\rho \\
\rho u \\
\rho v \\
\rho w \\
\rho E \\
p
\end{array}\right]_{\text {target }}=\left[\begin{array}{c}
\rho_{0}+\rho^{\prime} \\
0 \\
\left(\rho_{0}+\rho^{\prime}\right)\left(v^{\prime}\right) \\
0 \\
\left(p_{0}+p^{\prime}\right) /(\gamma-1)+0.5\left(\rho_{0}+\rho^{\prime}\right)\left(v^{\prime}\right)^{2} \\
p_{0}+p^{\prime}
\end{array}\right]
$$

We apply these source terms to the gas phase prior to solving the pressure corrector step in our numerical algorithm, and we use a sponge amplitude of $C_{\text {sponge }}=1 / \Delta t$.

For these simulations of a levitated droplet, the computational domain is a cube with length 24 $\mathrm{mm}$. There are 80 mesh cells in the $x$ and $z$ directions. The mesh is stretched in the $y$-direction 
within the sponge regions in order to reduce computational cost while allowing the sponge to span more of the acoustic wavelength. The sponge length is $7 \mathrm{~mm}$. Near the center of the domain, where the mesh is not stretched, the mesh dimension in $y$ is identical to the other directions. The timestep is $\Delta t=0.8 \mu \mathrm{s}$. This leads to an acoustic CFL of just less than unity in the gas phase but greater than 3 in the liquid phase. The stability of our numerical algorithm is unaffected by the acoustic CFL condition, so this choice allows us to balance the need to resolve the acoustic processes in the gas phase with the cost savings of a larger timestep. We use periodic boundary conditions in $x$ and $z$ while the sponges account for the boundaries in $y$. The gravitational acceleration is set to $-9.8 \mathrm{~m} / \mathrm{s}^{2}$ in the $y$-direction. To mimic the microphone placement in the experimental setup [23], we measure $p_{\mathrm{rms}}$ in a $1 \mathrm{~mm}$ diameter disk placed in the pressure antinode above the droplet. Figure 1 shows the arrangement of the simulation, with sponge zones and the notional microphone area.

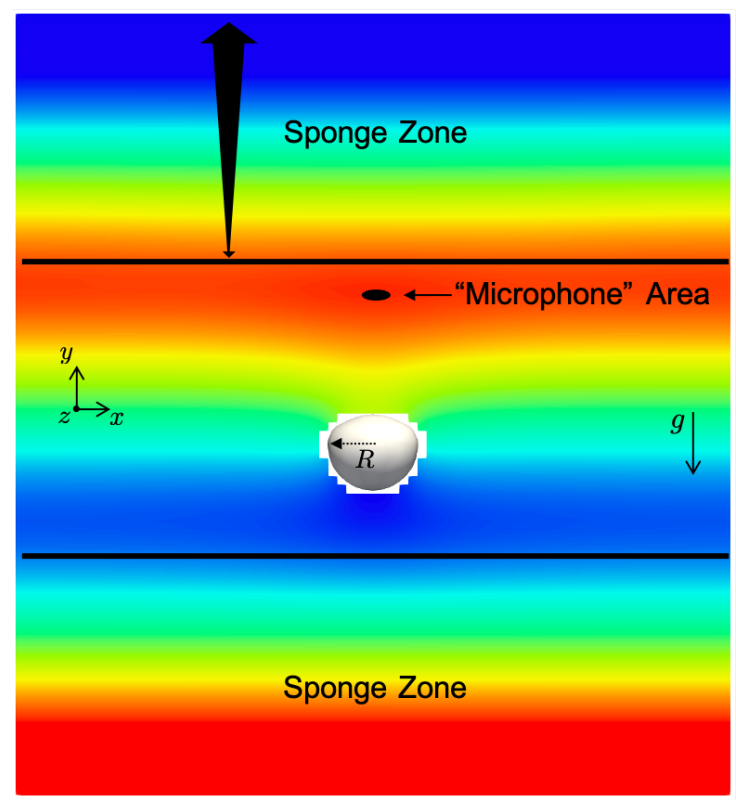

Figure 1. Diagram of simulation setup. Sponge zones are at the top and bottom of the domain. Gravity, $g$, acts downward, i.e., the negative $y$-direction. Colored white, the droplet is visualized with the 0.5 isocontour of volume fraction, and its deformed radius is indicated by $R$. Around the droplet, single-phase cells in the central $x y$-plane are colored by the gas pressure, with red corresponding to $104.9 \mathrm{kPa}$ and blue corresponding to $97.8 \mathrm{kPa}$. The "microphone" area is used to measure the RMS pressure, $p_{\mathrm{rms}}$, over the course of the simulations.

For the three cases that we test, we plot the acoustic bond number,

$$
B_{a}=\frac{2 p_{\mathrm{rms}}^{2} R_{s}}{\rho_{0} c_{0}^{2} \sigma}
$$

as a function of the normalized deformed droplet radius,

$$
R^{*}=\frac{R}{R_{s}},
$$

shown in figure 2, which also shows the deformed shapes of the droplets.

Our results closely match the experimental data, falling within the stated uncertainty of the measurements. As $B_{a}$ increases, the numerical $R^{*}$ increases more quickly than the reference. This could be due to the difference between the original experimental setup, which uses a single sound source and a reflector, and our simulation design, which has two sound sources and assumes a standing wave solution. The prescribed acoustic wave intensities are $A=$ $3.3 \mathrm{kPa}, 3.6 \mathrm{kPa}$, and $3.9 \mathrm{kPa}$ for the simulation data shown. These data were recorded when the droplet deformed radius and RMS pressure had come to steady-state. 


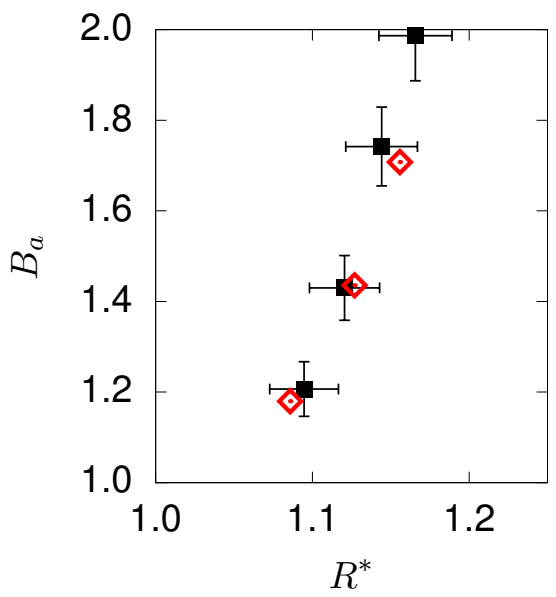

(a)

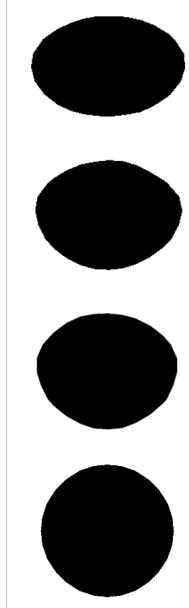

(b)

Figure 2. Droplet levitation data. (a) Acoustic Bond number $\left(B_{a}\right)$ as a function of the normalized deformed droplet radius $\left(R^{*}\right)$. The simulation points are in red, which are compared to reference points from [23]. (b) Droplet profiles at instance of measurement. The lowest picture is the initial shape, with each profile above it corresponding to an increase in acoustic Bond number.

\section{Results and discussion}

Having confirmed the ability of our numerical solver to capture the interactions between liquid droplets and strong acoustic perturbations through levitation, we now focus on acousticallydriven atomization of droplets. In these simulations, gravity is turned off, eliminating its effect on the droplet location. To better resolve the breakup process, the computational domain length is changed to $18 \mathrm{~mm}$ and the number of cells in each direction increased to 200 . This results in the initial droplet diameter being spanned by about 32 computational cells. The timestep is set to $0.25 \mathrm{\mu s}$. To promote atomization, the forced sound wave amplitude is $10 \mathrm{kPa}$. This means that the expected acoustic streaming velocity around the droplet is more than three times the critical value, according to the simple model given by Basu et al.[24].

Figure 3(a) shows the liquid-gas interface shortly after breakup has begun. The droplet is initialized at the pressure node, and as time progresses, the acoustic radiation force flattens the droplet. Before the inner surface of the droplet reaches a uniform thickness, the rim breaks from the droplet due to surface tension. At this resolution, it is unclear how much the limitations of the mesh cell length scale contribute to the surface tension effects present at the rim. In experiments on atomizing levitated droplets, such as $[25,26]$, the droplet has been shown to flatten to a uniform thickness prior to breakup, but these experiments were performed at significantly lower sound pressures. The accumulation of liquid at the rim is consistent with experiments and intuition, although the mesh might lack the resolution to capture fine azimuthal disturbances which have been shown to lead to the radial ejection of droplets [23].

By varying the initial position and acoustic frequency of the acoustic forcing, we can infer the impact of these attributes. Table 1 lists the properties of each case, with Case A unchanged, Case $B$ initialized at the pressure antinode, and Case $C$ using triple the frequency. We also test the effect of a turbulent setting by initializing homogeneous isotropic turbulence in the gas phase with a Taylor-scale Reynolds number of $\operatorname{Re}_{\lambda}=70$. In this instance, Case $\mathrm{D}$, the cell height in $y$ is halved near the center of the domain and the timestep is reduced to $0.125 \mu \mathrm{s}$.

Figure 3(b) shows that the droplet is extended vertically at the antinode, with the acoustic radiation force pulling liquid in opposite longitudinal directions, creating a stem shape. This continues until the edges of the elongated droplet reach pressure nodes, where the liquid is then pulled radially. As the rim breaks away and ligaments are formed, these ligaments retain momentum directed away from the pressure antinode, creating a bowl pattern instead of a simple disk. The formation of the stem structure is a novel atomization behavior, one not easily 
Table 1. Atomization simulations.

\begin{tabular}{c|rcc}
\hline Case & Frequency, $f(\mathrm{kHz})$ & Initial Location in $p$-wave & Initial Flowfield \\
\hline A & 21.76 & Node & Standing Wave \\
B & 21.76 & Antinode & Standing Wave \\
C & 65.28 & Node & Standing Wave \\
D & 21.76 & Node & Turbulence
\end{tabular}

accessible to earth-based experiments since it results from the placement of the droplet at the antinode. When the frequency is increased in Case $C$, the droplet experiences a combination of the phenomena observed in Case $\mathrm{A}$ and $\mathrm{B}$, since the initial droplet diameter spans a pressure node and two antinodes $\left(2 R_{s} / \lambda=0.55\right)$. As the droplet grows radially and flattens, it also elongates upward and downward. With some liquid moving longitudinally, the breakup at the rim occurs at a shorter radial distance and moves outward with less momentum than Case A.

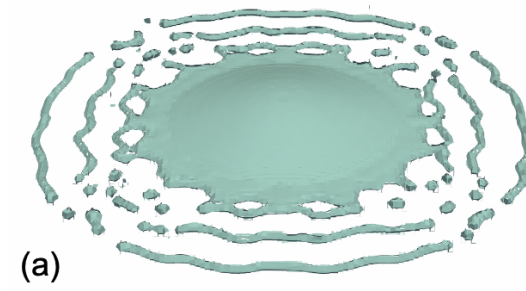

(c)

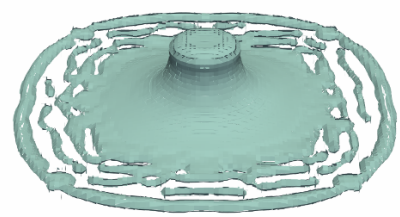

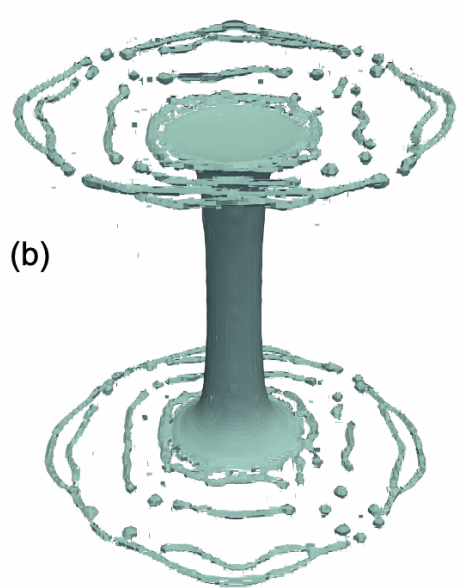

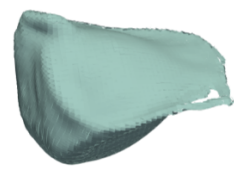

(d)

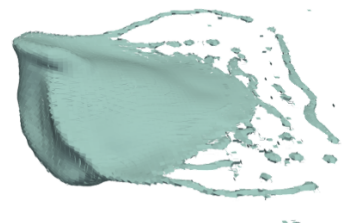

Figure 3. Liquid-gas interface (PLIC) shortly after breakup begins. (a) Case A at $2.4 \mathrm{~ms}$. (b) Case B at $8.7 \mathrm{~ms}$. (c) Case C at $2.8 \mathrm{~ms}$. (d) Case D at $1 \mathrm{~ms}$ (top) and $1.3 \mathrm{~ms}$ (bottom). Images (a)-(c) are at the same scale, and the images of $(d)$ are at a closer viewpoint.

Comparing the topology from these three scenarios, we can infer the influence of droplet position and acoustic frequency on the atomization profile. While a droplet near a pressure antinode will be prone to flattening and ejecting drops radially, a droplet near a pressure node can undergo significant lengthening and deformation prior to atomization. This behavior can prolong the onset of atomization as well as imparting the resulting droplets with momentum in the longitudinal direction. Depending on the size of the droplet relative to the acoustic wavelength, this could alter which instability is dominant in contributing to breakup, a cause for further study. The acoustic frequency controls the hydrodynamic lengthscale, which can lead to, as in Case $\mathrm{C}$, a droplet being exposed to pressure nodes and antinodes simultaneously. In addition to the shorter waves having a diminished effect on the liquid phase, higher frequencies can also reduce breakup by modifying the time-averaged flow field in this way. In case $D$, the turbulence breaks the symmetry of the droplet, which leads to the acoustically-induced flattening effect accelerating in some regions. As a result, we observe breakup at a much earlier time.

\section{Conclusions}

Through the use of a highly capable compressible, multiphase flow solver, we have explored unique aspects of acoustically induced atomization and demonstrated the influence of droplet position, sound frequency, and background turbulence. In addition to identifying flow behaviors related to acoustic interactions with fuel injection strategies, we have also validated our 
approach by simulating levitated droplets and comparing with experiments. As we plan to leverage this numerical tool to improve understanding of such interactions, further studies will aim for increased mesh resolution and flow scenarios more relevant to fuel injection, such as droplets traversing a sound wave and droplets exposed to both turbulence and acoustic forcing.

\section{Acknowledgements}

This material is based upon work supported by the National Science Foundation Graduate Research Fellowship under Grant No. DGE-1650441. This work was also sponsored in part by the Office of Naval Research (ONR) through the Multidisciplinary University Research Initiatives (MURI) Program, under grant number N00014-16-1-2617.

\section{Nomenclature}

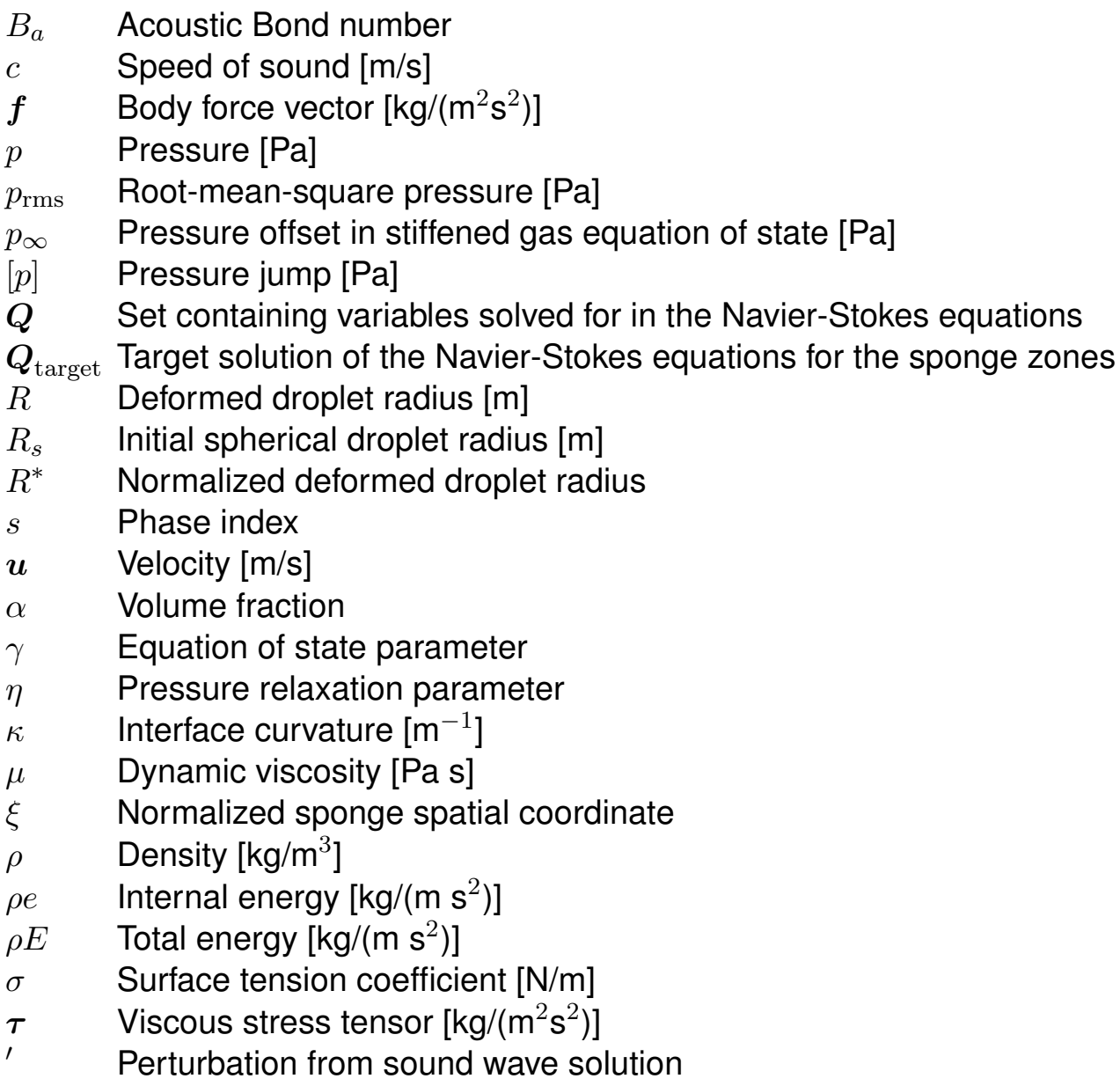

\section{References}

[1] Colin, O., Ducros, F., Veynante, D., and Poinsot, T., 2000, "A thickened flame model for large eddy simulations of turbulent premixed combustion," Physics of Fluids, 12(7), pp. 1843-1863.

[2] Baillot, F., Blaisot, J.-B., Boisdron, G., and Dumouchel, C., 2009, "Behaviour of an airassisted jet submitted to a transverse high-frequency acoustic field," J. Fluid Mech, 640, pp. 305-342.

[3] Narayanan, S., Srinivasan, K., and Sundararajan, T., 2013, "Atomization in the acoustic field of a Hartmann whistle," International Journal of Spray and Combustion Dynamics, 5(1), pp. 1-24.

[4] Brandt, E. H., 1989, "Levitation in physics," Science, 243(4889), pp. 349-355. 
[5] Lee, C. P., Anilkumar, A. V., and Wang, T. G., 1991, "Static shape and instability of an acoustically levitated liquid drop," Physics of Fluids A, 3(11), pp. 2497-2515.

[6] Ficuciello, A., Blaisot, J. B., Richard, C., and Baillot, F., 2017, "Investigation of air-assisted sprays submitted to high frequency transverse acoustic fields: Droplet clustering," Physics of Fluids, 29(6).

[7] Natarajan, M. and Desjardins, O., 2018, "Atomization in acoustically excited sprays," ICLASS 2018 - 14th International Conference on Liquid Atomization and Spray Systems.

[8] Lee, C. P., Anilkumar, A. V., and Wang, T. G., 1994, "Static shape of an acoustically levitated drop with wave-drop interaction," Physics of Fluids, 6(11), pp. 3554-3566.

[9] Di, W., Zhang, Z., Li, L., Lin, K., Li, J., Li, X., Binks, B. P., Chen, X., and Zang, D., 2018, "Shape evolution and bubble formation of acoustically levitated drops," Physical Review Fluids, 3(10), pp. 1-14.

[10] Yarin, A. L., Pfaffenlehner, M., and Tropea, C., 1998, "On the acoustic levitation of droplets," Journal of Fluid Mechanics, 356, pp. 65-91.

[11] Yarin, A. L., Brenn, G., Kastner, O., Rensink, D., and Tropea, C., 1999, "Evaporation of acoustically levitated droplets," Journal of Fluid Mechanics, 399, pp. 151-204.

[12] De Lorenzo, M., Pelanti, M., and Lafon, P., 2018, "HLLC-type and path-conservative schemes for a single-velocity six-equation two-phase flow model: A comparative study," Applied Mathematics and Computation, 333, pp. 95-117.

[13] Kuhn, M. and Desjardins, O., "An all-Mach, low-dissipation strategy for simulating multiphase flows," , Journal of Computational Physics, Under Review.

[14] Owkes, M. and Desjardins, O., 2014, "A computational framework for conservative, threedimensional, unsplit, geometric transport with application to the volume-of-fluid (VOF) method," Journal of Computational Physics, 270, pp. 587-612.

[15] Owkes, M. and Desjardins, O., 2017, "A mass and momentum conserving unsplit semiLagrangian framework for simulating multiphase flows," Journal of Computational Physics, 332, pp. 21-46.

[16] Subbareddy, P. K. and Candler, G. V., 2009, "A fully discrete, kinetic energy consistent finite-volume scheme for compressible flows," Journal of Computational Physics, 228(5), pp. 1347-1364.

[17] Youngs, D. L., 1982, "Time-Dependent Multi-Material Flow with large Fluid Distorsion." Numerical Methods in Fluid Dynamics, pp. 273-285.

[18] Pilliod, J. E. and Puckett, E. G., 2004, "Second-order accurate volume-of-fluid algorithms for tracking material interfaces," Journal of Computational Physics, 199(2), pp. 465-502.

[19] Chiodi, R. and Desjardins, O., "General, Robust, and Efficient Polyhedron Intersection in the Interface Reconstruction Library," , Journal of Computational Physics, Under Review.

[20] Chiodi, R., "Gitlab: Interface Reconstruction Library," . https://gitlab.com/ robertchiodi/interfacereconstructionlibrary

[21] Chiodi, R. M., 2020, "Advancement of Numerical Methods for Simulating Primary Atomization," Ph.d., Cornell University.

[22] Kwatra, N., Su, J., Grétarsson, J. T., and Fedkiw, R., 2009, "A method for avoiding the acoustic time step restriction in compressible flow," Journal of Computational Physics, 228(11), pp. 4146-4161.

[23] Anilkumar, A. V., Lee, C. P., and Wang, T. G., 1992, "Stability of an acoustically levitated and flattened drop: An experimental study," Physics of Fluids A, 5(11), pp. 2763-2774.

[24] Basu, S., Saha, A., and Kumar, R., 2012, "Thermally induced secondary atomization of droplet in an acoustic field," Applied Physics Letters, 100(5).

[25] Aoki, K. and Hasegawa, K., 2020, "Acoustically induced breakup of levitated droplets," AIP Advances, 10(5).

[26] Naka, M. and Hasegawa, K., 2020, "Breakup characteristics of levitated droplets in a resonant acoustic field," Physics of Fluids, 32(12), p. 124109. 